\title{
Les ricochets
}

Christophe Clanet ${ }^{1}$ et Lydéric Bocquet ${ }^{2}$

1. Institut de Recherche sur les Phénomènes Hors Équilibre, UMR CNRS 6594, Marseille 2. Laboratoire PMCN, UMR CNRS 5586, Université Lyon-I, Villeurbanne

Parmi les questions physiques associées aux ricochets, nous essayons d'en traiter deux :

- sous quelles conditions un galet peut-il rebondir sur l'eau?

- combien de fois peut-il le faire?

Dans un premier temps, nous répondons expérimentalement à ces questions, en montrant que le rebond n'est possible que si l'orientation du galet est stabilisée de façon gyroscopique, et que ce rebond ne se produit qu'au-delà d'une vitesse d'impact minimale. Une modélisation théorique est ensuite proposée, permettant de comprendre ces propriétés et aussi d'identifier la source de dissipation responsable de la fin des ricochets.

\section{Introduction}

Remplaçons, l'espace de cet article, le « fais pas ci, fais pas ça » par le « pourquoi ci, pourquoi ça », hein ? Pourquoi pas?

Nous voici donc sur les bords du lac Victoria avec HumHum, notre ancêtre pêcheur, il y a 3,5 millions d'années :

HumHum : Pourquoi pas essayer pêcher en lançant cailloux sur poissons, hein Maman?

Lucie : Fais pas ça, HumHum.

Évidemment HumHum a essayé, mais plutôt que d'améliorer les techniques de pêche il a découvert un drôle de petit phénomène : les cailloux qui rebondissent.

HumHum : ??? (bouche bée)

Voici donc l'histoire vraie et inventée de l'origine des ricochets.

«Un, deux, trois, quatre » : c'est le nombre de ricochets réalisés sur la figure 1 .

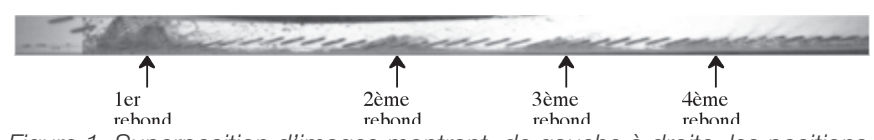

Figure 1. Superposition d'images montrant, de gauche à droite, les positions successives du galet (en forme de disque vu par la tranche) au cours de ses quatre rebonds. Les quatre monticules grisés sont les éclaboussures de l'eau générées aux points d'impact du galet.

Les règles du jeu n'ont jamais changé [1] : un galet ou un coquillage est lancé à la surface de l'eau et le nombre de rebonds distingue le vainqueur (le record du monde actuel est de 40 rebonds [2]). Parmi les questions associées à ce jeu, nous essayons d'en traiter deux dans cet article:

- pourquoi un galet rebondit-il sur l'eau ?

- combien de fois peut-il le faire ?

En notant $\mathrm{R}$ le rayon du galet, $\mathrm{m}$ sa masse et $\mathrm{U}$ sa vitesse, son énergie à l'impact est essentiellement cinétique $\left(\mathrm{mU}^{2} /(\mathrm{mgR}) \gg 1\right)^{1}$. A priori, le travail des forces susceptibles de modifier la trajectoire rectiligne du galet peut être d'origine inertielle $\left(\rho R^{3} U^{2}\right.$, où $\rho$ est la densité de l'eau), visqueuse ( $\mu \mathrm{UR}^{2}$, où $\mu$ est la viscosité dynamique de l'eau) ou capillaire $\left(\sigma R^{2} \text {, où } \sigma \text { est la tension de surface de l'eau }\right)^{2}$. Les nombres sans dimension qui comparent ces effets entre eux sont respectivement le nombre de Reynolds $R_{e} \equiv \rho U R / \mu \geq 10^{4}$ et le nombre de Weber $W_{e} \equiv \rho U^{2} R / \sigma \geq 10^{3}$. Ainsi, notre étude est menée dans la limite des grands nombres de Reynolds et des grands nombres de Weber, où la force de réaction de l'eau d'origine inertielle domine les forces visqueuses et capillaires. Dans cette limite, la force qui s'oppose à la trajectoire recti-

1. $\mathrm{mU}^{2} /(\mathrm{mgR}) \approx 50$ pour $\mathrm{U} \approx 3 \mathrm{~m} / \mathrm{s}, \mathrm{g} \approx 10 \mathrm{~m} / \mathrm{s}^{2}, \mathrm{R} \approx 2 \mathrm{~cm}$.

2. Les effets inertiels correspondent à l'énergie cinétique de l'eau déplacée, les effets visqueux à la résistance éprouvée par le galet se déplaçant dans le fluide visqueux et les effets capillaires à l'augmentation de l'interface eau-air lorsque le galet pénètre dans l'eau et crée une cavité.
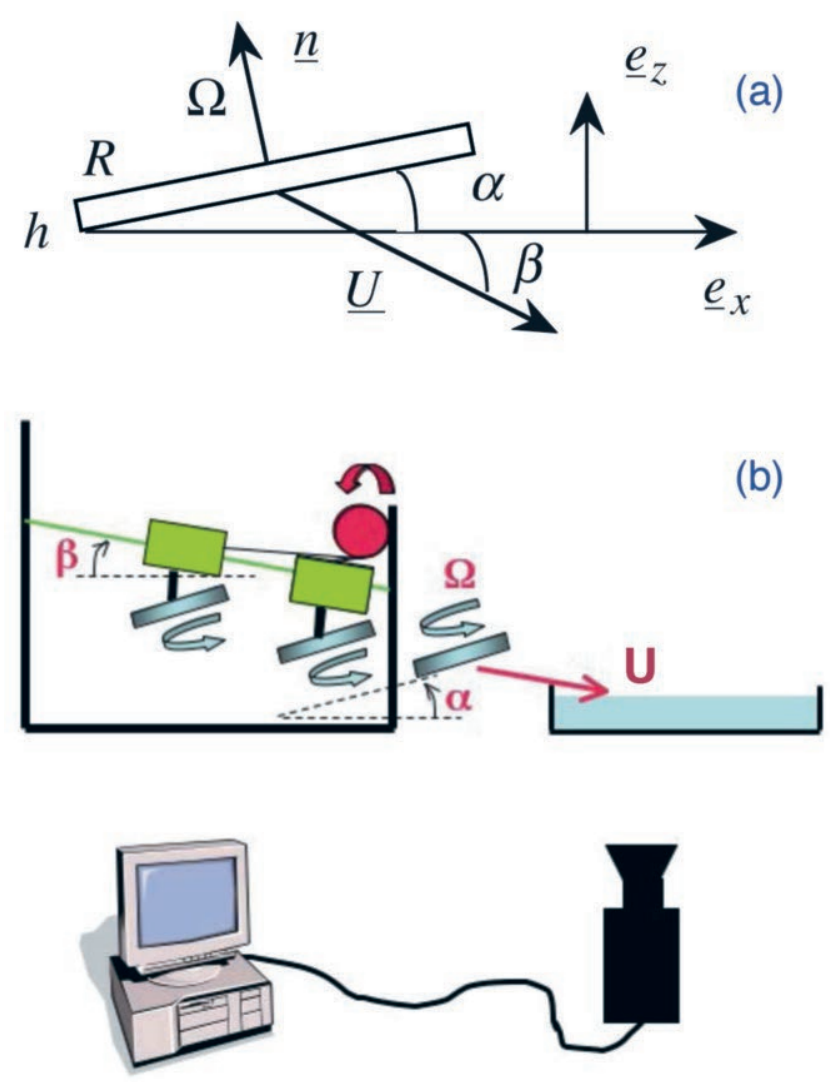

Figure 2. (a) Conventions utilisées. (b) Schéma de principe du dispositif de "Ricoche » (notre montage expérimental).

ligne du galet est la force de réaction associée au déplacement de l'eau.

Dans cette limite, quelques approches théoriques ont déjà essayé de répondre aux questions posées $[3,4,5]$, mais sans aucune donnée expérimentale. Nous reviendrons sur ces approches à la lumière de nos résultats expérimentaux $[6,7]$.

\section{Dispositif expérimental}

Les conventions utilisées sont présentées sur la figure 2(a) : le caillou est un disque d'aluminium d'épaisseur $h$ et de rayon $R$. Sa vitesse de translation est notée $\boldsymbol{U}$ et sa vitesse de rotation $\boldsymbol{\Omega}=\boldsymbol{\Omega} . \boldsymbol{n}$, où $\boldsymbol{n}$ est le vecteur unitaire normal à sa surface. L'orientation du galet est définie par l'angle d'attaque $\alpha$ tel que $\cos \alpha=\boldsymbol{n} . \boldsymbol{e}_{\mathbf{z}}$, où $\boldsymbol{e}_{\boldsymbol{z}}$ est le vecteur unité normal à la surface non perturbée de l'eau. La direction du mouvement du galet est définie par l'angle $\beta$ tel que $\cos \beta=\boldsymbol{U} \cdot \boldsymbol{e}_{\boldsymbol{x}}$, où $\boldsymbol{e}_{\boldsymbol{x}}$ est le vecteur unité parallèle à la surface de l'eau. 
Le schéma du montage expérimental " Ricoche » est présenté sur la figure 2(b). Ce montage permet de contrôler indépendamment $\Omega, \mathrm{U}, \alpha$ et $\beta$ : le galet est tenu par dépression à un boîtier contenant un moteur électrique permettant de contrôler la vitesse de rotation $\Omega$. Ce boîtier translate à la vitesse $U$ sur deux rails dont l'inclinaison par rapport à la surface horizontale de l'eau est $\beta$. Enfin, l'inclinaison du boîtier par rapport aux rails permet de régler $\alpha$ (indépendamment de $\beta)$. Le détachement du galet est assuré par des contacteurs électriques qui actionnent une électrovanne qui relâche la dépression. Le galet poursuit alors sa trajectoire jusqu'au point d'impact.

L'impact du galet avec la surface est enregistré avec une caméra vidéo rapide (Kodak HS4540). La plupart des expériences sont menées avec un disque d'aluminium (densité $\rho_{\mathrm{S}}$ ), soit un rapport de densité : $\rho_{S} / \rho \cong 2,7$. Les propriétés géométriques des cailloux utilisés sont rappelées dans le tableau 1. L'ordre de grandeur des vitesses est $1 \mathrm{~m} / \mathrm{s}<U<10 \mathrm{~m} / \mathrm{s}$ et les vitesses de rotation varient entre $0 \mathrm{rad} / \mathrm{s}$ et $600 \mathrm{rad} / \mathrm{s}$.

\begin{tabular}{|c|c|c|c|}
\hline Disque & $\mathrm{R}(\mathrm{mm})$ & $\mathrm{h}(\mathrm{mm})$ & $\mathrm{m}(\mathrm{g})$ \\
\hline 1 & 25 & 2,75 & 15 \\
\hline 2 & 25 & 5,55 & 30 \\
\hline 3 & 50 & 2,75 & 57 \\
\hline 4 & 50 & 5,55 & 120 \\
\hline
\end{tabular}

Tableau 1. Dimensions et masses des disques en aluminium utilisés.

\section{Résultats expérimentaux}

Un exemple de ricochet est présenté sur la figure 3 : en notant $\mathrm{t}=0$ l'instant où le galet touche la surface et $\mathrm{t}=\tau$ celui où il en sort, on mesure sur la figure 3 un temps de contact $\tau \cong 32 \mathrm{~ms}$. On observe sur cette chronophotographie que l'angle d'attaque $\alpha$ reste constant durant tout l'impact. Comme nous le verrons, ceci est le signe d'une forte stabilisation " gyroscopique " (liée à la rotation du galet sur luimême). On remarque aussi que la cavité créée par le galet
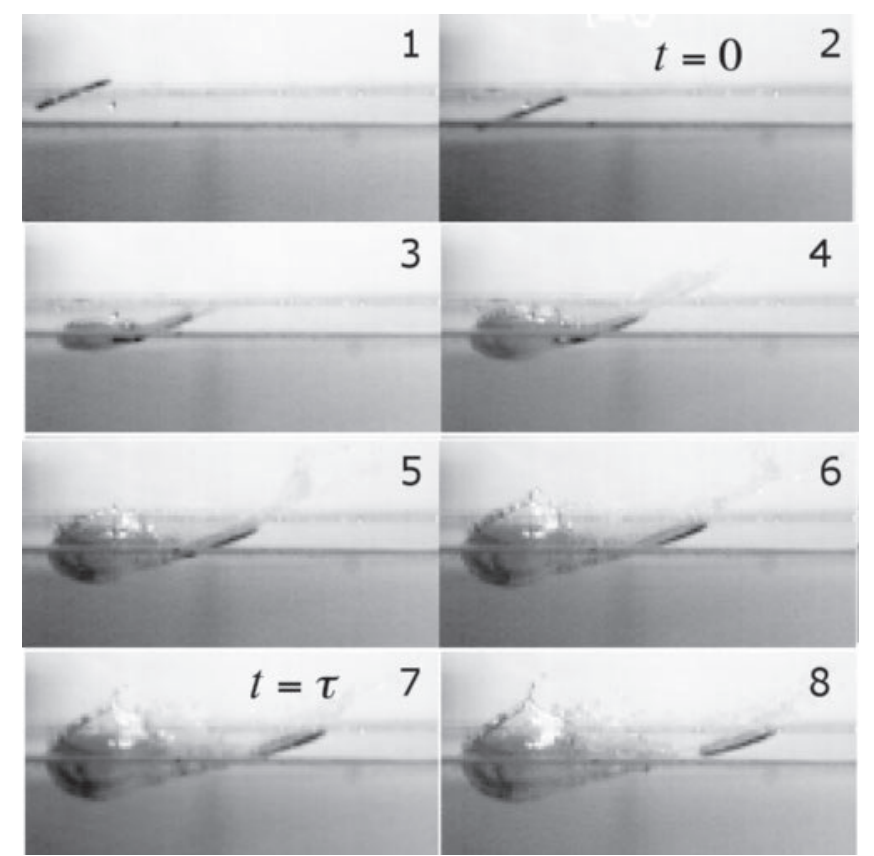

Figure 3. Chronophotographie d'un ricochet obtenue avec le galet 1 et $U=3,5 \mathrm{~m} / \mathrm{s}, \Omega=65 \mathrm{rot} / \mathrm{s}, \alpha=20^{\circ}, \beta=20^{\circ}$. Le temps s'écoule de gauche à droite et de haut en bas avec le pas $\Delta t=6,5 \mathrm{~ms}$.
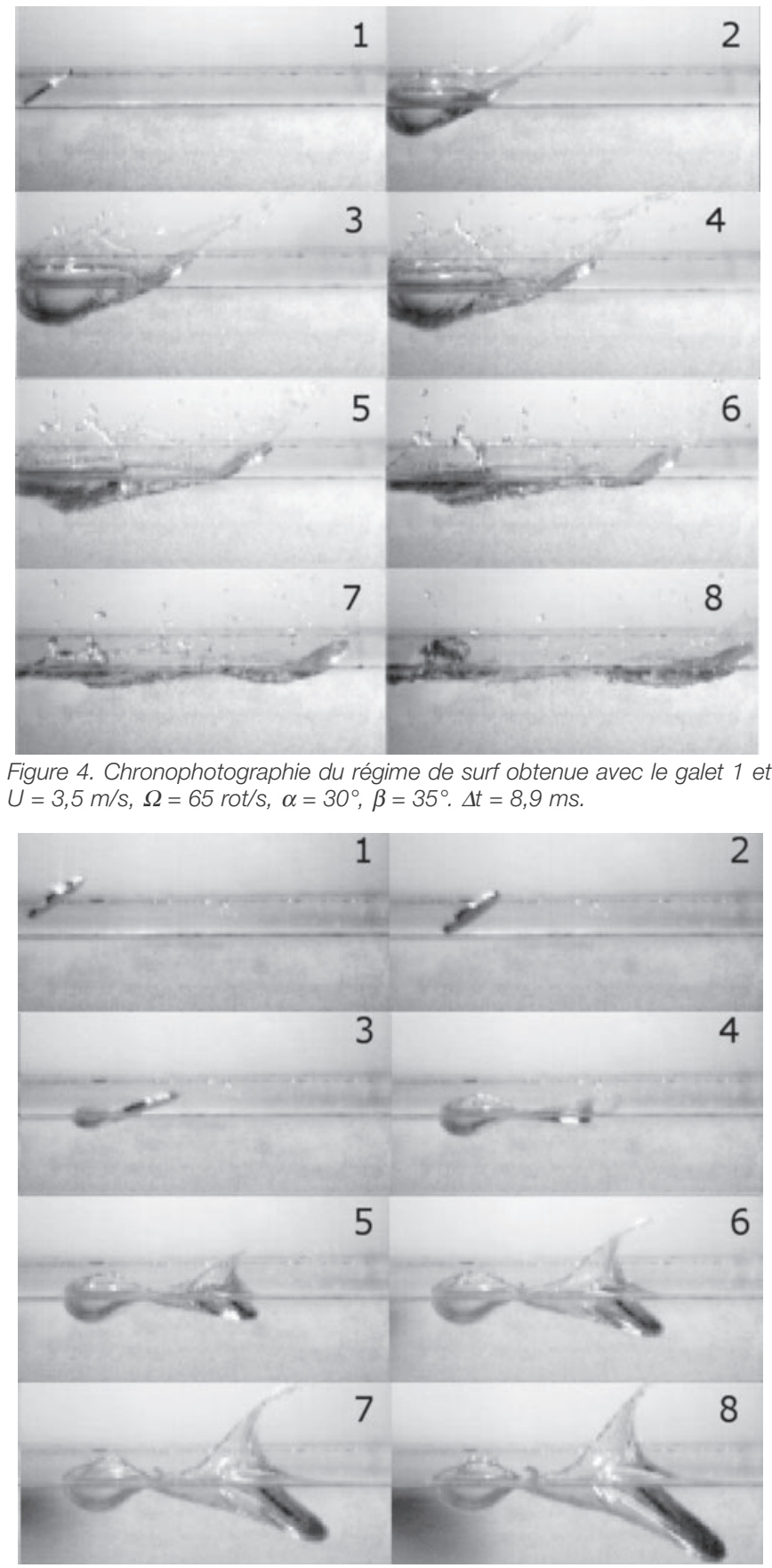

Figure 5. Chronophotographie illustrant l'effet de la rotation pour un galet 1 et $U=3,5 \mathrm{~m} / \mathrm{s}, \Omega=0 \mathrm{rot} / \mathrm{s}, \alpha=35^{\circ}, \beta=20^{\circ} . \Delta t=8,9 \mathrm{~ms}$.

n'est pas symétrique : sa courbure est plus importante côté entrée que côté sortie.

Les rebonds se succèdent de façon « auto-similaire » jusqu'à ce que les paramètres d'impact (angles et vitesse) ne permettent plus au galet de s'extraire de l'eau. Le galet " surfe " alors à la surface, comme le montre la figure 4. Ainsi, la fin des ricochets n'est pas caractérisée par un enfoncement gravitaire (qui viendra plus tard) mais par un régime de surf, où le galet reste à la surface avec un angle d'incidence constant mais ne ressort plus de l'eau.

L'effet de la rotation est de stabiliser l'orientation du galet (angle $\alpha$ ) de façon gyroscopique [5]. Cet effet est illustré sur la figure 5 , où $\Omega=0$ rot/s : sans rotation, le galet culbute à l'impact et plonge.

De façon plus quantitative, la figure 6(a) présente l'évolution du temps de contact $\tau$ en fonction de $\Omega$. Dans la limite des 

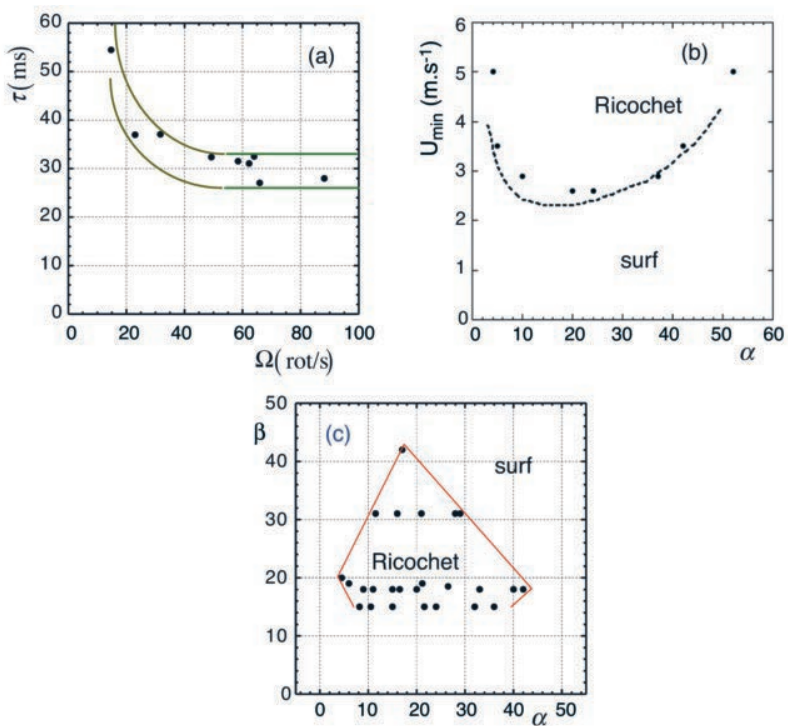

Figure 6. (a) Évolution du temps de contact mesuré $\tau$ en fonction de $\Omega$ pour le galet 1, avec $U=3,5 \mathrm{~m} / \mathrm{s}, \alpha=20^{\circ}, \beta=20^{\circ}$. (b) Domaine d'existence des ricochets dans le plan $\left\{\alpha, \cup_{\min }\right\}$ pour le galet 1 et $\Omega=65 \mathrm{rot} / \mathrm{s}, \beta=20^{\circ}$. Les pointillés représentent la prédiction théorique. (c) Domaine d'existence des ricochets dans le plan $\{\alpha, \beta\}$ pour le galet 1 et $\Omega=65 \mathrm{rot} / \mathrm{s}, U=3,5 \mathrm{~m} / \mathrm{s}$. Dans les figures a et $c$, les lignes continues sont des guides pour les yeux.

faibles rotations, le temps de contact diverge pour cause de non-rebond et dans la limite des fortes rotations, il tend vers une limite, ici de l'ordre de $30 \mathrm{~ms}$.

Sans entrer dans le détail de la stabilisation à l'impact, on peut comparer le temps de contact $\tau$ au temps $1 / \Omega$ caractérisant la rotation. Sur la figure $6(\mathrm{a}), \Omega \tau \geq 1$ pour $\Omega \geq 40 \mathrm{rot} / \mathrm{s}$. On observe sur cette figure que le galet est stabilisé lorsqu'il a le temps de faire plus d'un tour sur lui-même pendant l'impact. Dans le reste de l'article, nous ne considérons que le cas $\Omega \tau \geq 1$, où $\alpha$ est constant durant l'impact.

Dans la limite $\Omega \tau \gg>1$ et avec les trois paramètres de contrôle restants $\{U, \alpha, \beta\}$, on peut construire un diagramme de phases indiquant les domaines d'existence des ricochets. Des coupes de ce diagramme dans les plans $\{U, \alpha\}$ et $\{\alpha, \beta\}$ sont présentées sur les figures $6(\mathrm{~b})$ et $6(\mathrm{c})$.

De façon assez inattendue, la valeur $\alpha \cong 20^{\circ}$ joue un rôle particulier : la vitesse minimale, $U_{\min }$, au-delà de laquelle le galet rebondit $\left(U_{\min } \cong 2,6 \mathrm{~m} / \mathrm{s}\right)$ est obtenue pour $\alpha \cong 20^{\circ}$ [figure 6(b)]. De même, le domaine le plus étendu en $\beta$ est obtenu pour $\alpha \cong 20^{\circ}$ [figure $6(\mathrm{c})$ ]. Dans les régions de non-rebond, le galet ne culbute pas, puisqu'il est ici toujours stabilisé par rotation, mais il surfe, comme présenté sur la figure 4.
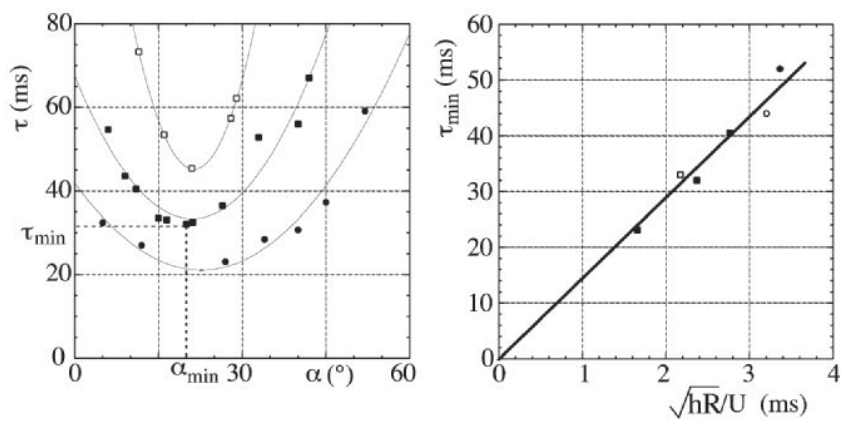

Figure 7 : (a) Évolution du temps de contact $\tau$ en fonction de $\alpha$ pour le galet 1 et différentes conditions: (carrés pleins) $U=3,5 \mathrm{~m} / \mathrm{s}, \beta=20^{\circ}$, (carrés vides) $U=3,5 \mathrm{~m} / \mathrm{s}, \beta=30^{\circ}$, (cercles pleins) $U=5 \mathrm{~m} / \mathrm{s}, \beta=20^{\circ}$. (b) Évolution du temps de contact minimal $\tau_{\min }$ en fonction du temps caractéristique $(h R)^{1 / 2} / \mathrm{U}$ pour $\beta=20^{\circ}$ et $\alpha=20^{\circ}$ et différents galets : (carrés pleins) $R=2,5 \mathrm{~cm}$, $h=2,75 \mathrm{~mm}$. (carrés vides) $R=5 \mathrm{~cm}, h=2,75 \mathrm{~mm}$, (cercles pleins) $R=2,5 \mathrm{~cm}$, $h=5,55 \mathrm{~mm}$, (cercles vides) $R=5 \mathrm{~cm}, h=5,55 \mathrm{~mm}$. La ligne représente le résultat obtenu par intégration numérique de l'équation (2).
Concernant le temps de contact $\tau$, la figure 7(a) montre que pour une vitesse et un angle $\beta$ donnés, le temps de contact est minimal pour la même valeur $\alpha \cong 20^{\circ}$. Le temps de contact minimal est noté $\tau_{\min }$. Son évolution avec la vitesse $U$ et pour les différents galets est présentée sur la figure 7(b). On observe que le temps de contact minimal suit la loi d'échelle $\tau_{\min } \propto$ $(\mathrm{hR})^{1 / 2} / \mathrm{U}$ (ces résultats sont obtenus avec $\alpha \cong 20^{\circ}$ et $\beta \cong 20^{\circ}$ ).

Cette relation peut-être retrouvée par analyse dimensionnelle : en notant $F_{L}$ la force de réaction de l'eau sur le galet, le temps de contact peut-être évalué à partir de la loi de Newton comme $\tau \propto\left(\mathrm{mR} / \mathrm{F}_{1}\right)^{1 / 2}$ où $\mathrm{m}$ est la masse du galet. À grand nombre de Reynolds, $F_{L} \propto \rho S_{\text {wetted }} U^{2}$ (produit de la masse de liquide déplacé et de son accélération), où $S_{\text {wetted }}$ est la surface mouillée $[8,9]$. Ainsi, avec $S_{\text {wetted }}=\pi R^{2}$ et $m=\rho_{S} \pi R^{2} h$, on retrouve le comportement : $\tau \propto(\mathrm{hR})^{1 / 2} / \mathrm{U}$.

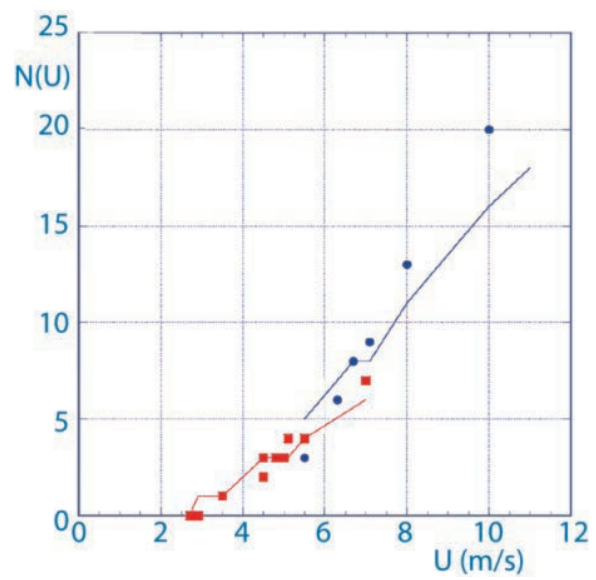

Figure 8 : Évolution du nombre de rebonds en fonction de la vitesse d'impact, pour le galet 1 et deux conditions initiales différentes, $\alpha=7^{\circ}$ et $\beta=20^{\circ}$ (carrés) et $\alpha=10^{\circ}$ et $\beta=9^{\circ}$ (cercles). Les lignes continues sont les prédictions théoriques [équation (2)] obtenues avec ces conditions initiales.

Concernant le nombre de rebonds, la figure 8 présente l'évolution du nombre de rebonds en fonction de la vitesse d'impact. Dans notre fenêtre d'observation, ce nombre est essentiellement une fonction linéaire de la vitesse au-delà d'une vitesse critique (ici de l'ordre de $3 \mathrm{~m} / \mathrm{s}$ ) en deçà de laquelle le galet surfe sans rebondir [voir figure 6(b)]. Nous montrons dans la partie théorique que la source de dissipation responsable de la fin des rebonds est la mise en mouvement du liquide par le galet à chaque impact. Comme nous le verrons, ce transfert d'impulsion galet/liquide se traduit comme une friction sur la dynamique du galet.

\section{Modèle théorique}

\section{Force de réaction}

La pierre angulaire du modèle est l'expression de la force exercée par le fluide sur le galet au cours de l'impact. Dans le régime des grands nombres de Reynolds $\left(R_{e} \equiv \rho U R / \mu \cong 10^{5}\right)$, les effets inertiels dominent les effets visqueux et la force exercée sur le galet est de la forme :

$\boldsymbol{F}_{L}=\mathrm{C}_{L} . \rho . \mathrm{S}_{\text {wetted }} \mathrm{f}(\alpha, \beta) . \mathrm{U}^{2} . \boldsymbol{n}$, où $\mathrm{C}_{\mathrm{L}}$ est un coefficient d'ordre unité et $\mathrm{f}(\alpha, \beta)$ une fonction adimensionnée qui contient la dépendance angulaire de la force.

De façon à déterminer $f(\alpha, \beta)$, nous avons mené des mesures de «portance » dans un canal hydraulique. Ces mesures sont bien prises en compte par l'expression :

$$
\boldsymbol{F}_{\mathbf{L}}=\mathrm{C}_{\mathrm{L}} \cdot \rho \cdot \mathrm{S}_{\text {wetted }} \cdot \sin (\alpha+\beta) \cdot \mathrm{U}^{2} \cdot \boldsymbol{n}
$$

La structure physique de cette force (en $\left.\rho . S_{\text {wetted. }} U^{2}\right)$ peut être comprise en termes d'impulsion communiquée par le galet au liquide par unité de temps : en effet, lorsqu'il rentre 
dans l'eau, le galet cherche à aller tout droit par inertie, mais pour cela il est obligé de déplacer du fluide : le volume déplacé par unité de temps est $S_{\text {wetted }} \cdot \sin (\alpha+\beta)$.U. L'impulsion communiquée à l'eau est donc par unité de temps : $\rho . S_{\text {wetted }} \sin (\alpha+\beta) . U^{2}$. Par réaction, la force exercée par l'eau sur le galet prend la forme présentée dans l'équation (1).

\section{Quelques remarques qualitatives}

Plusieurs remarques peuvent être faites en considérant la limite $\alpha<<1, \beta<<1$. Cette limite est en général atteinte après le premier impact (voir figure 1). Dans cette limite, la force exercée sur le galet le long de l'axe $x$ est :

$F_{x} \cong-0,5 . \rho . U^{2} \cdot S_{\text {wetted }}(\alpha+\beta) \cdot \alpha$. On en déduit que $F_{x} \propto \alpha^{2}$, ce qui implique que la variation de l'impulsion suivant $x$ est «lente » (ordre 2 en $\alpha$ ).

Suivant l'axe $z, F_{z} \cong-0,5 . \rho . U^{2} \cdot S_{\text {wetted }}(\alpha+\beta)$, d'où l'on déduit que $F_{z}$ est d'ordre 1 en $\alpha$. La variation de l'impulsion suivant $z$ est donc beaucoup plus rapide que suivant $x$. De plus, la dépendance en $\alpha+\beta$ implique qu'à une position $z$ fixée, $F_{z}$ est plus grande dans la phase descendante $(\beta>0)$ que dans la phase ascendante du galet $(\beta<0)$. Cette différence permet de comprendre la dissymétrie observée sur la forme de la cavité d'air creusée par le galet à l'impact (figure 3).

Enfin, concernant la stabilisation gyroscopique, les équations d'Euler pour les toupies symétriques [5] permettent d'évaluer la variation relative de l'angle d'incidence à l'impact sous la forme : $\delta \alpha / \alpha \propto F_{1} \cdot R /\left(m R^{2} \Omega^{2}\right)$. En utilisant l'expression de la force $F_{L}$, on obtient : $\delta \alpha / \alpha \propto \rho / \rho_{S} . U^{2} /\left(R h \Omega^{2}\right)$. La stabilisation du galet $(\delta \alpha / \alpha<<1)$ est donc obtenue dans la limite $\mathrm{Rh} \Omega^{2} / \mathrm{U}^{2}>>\rho / \rho_{\mathrm{S}}$, soit encore $\Omega \tau>>\left(\rho / \rho_{\mathrm{S}}\right)^{1 / 2}$.

\section{Équations du mouvement}

L'équation qui décrit le mouvement du galet stabilisé gyroscopiquement est :

$$
\mathrm{m}(\mathrm{d} \boldsymbol{U} / \mathrm{dt})=\mathrm{K} \cdot 0,5 \cdot \rho \cdot \mathrm{U}^{2} \cdot \mathrm{S}_{\text {wetted }} \cdot \sin (\alpha+\beta) \cdot \boldsymbol{n}+\mathrm{m} \cdot \boldsymbol{g}
$$

où $U^{2}=U_{x}{ }^{2}+U_{z}^{2}, g$ est l'accélération de la pesanteur et la constante $\mathrm{K}$ permet de décrire le mouvement dans l'eau $(K=1)$ et dans l'air $(K=0)$. Les conditions initiales qui doivent être satisfaites par la solution de l'équation (2) sont :

$$
\mathrm{U}=\left(\mathrm{U}_{\mathrm{x}}{ }^{2}(\mathrm{t}=0)+\mathrm{U}_{\mathrm{z}}{ }^{2}(\mathrm{t}=0)\right)^{1 / 2}, \beta=\operatorname{Arctan}\left[-\mathrm{U}_{\mathrm{z}}(\mathrm{t}=0) / \mathrm{U}_{\mathrm{x}}(\mathrm{t}=0)\right] \text {. }
$$

Par convention, $z(t=0)=0$. L'équation non linéaire (2) est intégrée numériquement.

Le premier résultat concerne l'existence d'une vitesse minimale $U_{\min }$ pour observer le rebond du galet. Mathématiquement, $U_{\text {min }}$ est définie comme la vitesse minimale au-delà de laquelle l'équation $\mathrm{z}(\mathrm{t})=0$ admet une solution non nulle. On présente sur la figure $6(b)$ la vitesse minimale ainsi obtenue. Malgré la simplicité du modèle, cette figure montre un bon accord avec les résultats expérimentaux.

II est aussi possible d'obtenir numériquement le nombre total de rebonds pour une vitesse et des angles donnés. Après la collision $\mathrm{N}$, la vitesse $\mathrm{U}$ et l'angle $\beta$ sont calculés au temps $t=\tau$. Ces valeurs sont ensuite utilisées comme valeurs d'entrée pour la collision $\mathrm{N}+1$. Ce processus est répété jusqu'à ce que le galet ne sorte plus de l'eau. Les prédictions ainsi obtenues sont présentées sur la figure 8. L'accord avec les résultats expérimentaux est encore satisfaisant.

\section{Source de dissipation}

On se concentre à présent sur la source de dissipation, responsable de l'arrêt des rebonds. Cette discussion repose sur les observations suivantes : la composante horizontale de la vitesse varie peu au cours d'une collision (en particulier dans la limite $\alpha<<1$ ), tandis que l'angle $\beta \equiv \operatorname{Arctan}\left[-U_{z}(t) / U_{x}(t)\right]$ dimi- nue au fil des collisions. En faisant l'approximation $U_{x}(t)=U$, on obtient pour des petits angles : $\beta \cong-U_{z}(t) / U$. Ces observations permettent de développer l'expression de la force en série de Taylor en $\alpha$ et $\beta$, et de simplifier l'équation du mouvement selon z :

$$
\mathrm{m}\left(\mathrm{dU}_{\mathrm{z}} / \mathrm{dt}\right)=\mathrm{F}^{(0)}(\mathrm{z})-\zeta(\mathrm{z}) \mathrm{U}_{\mathrm{z}}-\mathrm{mg}
$$

où $F(0) \cong 0,5 \cdot \rho \cdot U^{2} \cdot S_{\text {wetted }}(z) \cdot \sin (\alpha) \cdot \cos (\alpha)$ et $\zeta(z) \cong$ 0,5.p.U $U^{2} \cdot S_{\text {wetted }}(z) \cdot \cos ^{2}(\alpha)$. Le paramètre $\zeta(z)$ joue le rôle d'un coefficient de friction.

On reconnaît dans (3) l'équation du mouvement d'un oscillateur non linéaire amorti. Ceci montre que la vitesse suivant $z$ (et donc $\beta$ ) va diminuer au fil des collisions.

\section{Conclusion}

Nous avons étudié la physique des ricochets dans la limite des fortes vitesses de rotation. Nous avons montré que dans ce régime, les galets rebondissent sous l'effet de la réaction de l'eau et que la source de dissipation réside dans la dépendance de cette réaction à la direction de la vitesse.

L'influence de la forme du galet n'est pas traitée dans notre travail et peut être considérée comme une extension possible, tout comme l'étude des rebonds dans un régime de rotation intermédiaire $\Omega \tau$ d'ordre unité.

\section{Remerciements}

Nous avons mené cette étude essentiellement pour répondre à la curiosité de nos enfants. Nous ne sommes pas certains que la réponse les passionne pour le moment (le plus grand a 11 ans). Le problème a cependant intrigué (voire passionné) deux étudiants très enthousiastes, $L$. Rosellini et $F$. Hersen, sans qui nous aurions avancé beaucoup moins vite. Enfin, ce travail n'aurait pas abouti sans les efforts techniques de F. Dutertre et J. Minelli. Merci à tous pour cette conviviale et intéressante aventure.

\section{Références}

[1] D'Arcy Thomson (2000), Alliage, 44, 77-78.

[2] Le record du monde actuel semble être de 40 rebonds. II est détenu par Kurt Steiner. Plus d'informations sont disponibles sur le site http://www.stoneskipping.com

[3] C.L. Stong (1968), "The Amateur Scientist", Sci. Amer., 219, 112118.

[4] H.R. Crane (1988), "How things work: What can a dimple do for skipping stones?", Phys. Teach., 26, 300-301.

[5] L. Bocquet (2003), "The Physics of Stone Skipping", American Journal of Physics, 71, 150.

[6] C. Clanet, F. Hersen and L. Bocquet (2004), "Secrets of successful stone-skipping", Nature, 427, 29.

[7] L. Rosellini, F. Hersen, C. Clanet and L. Bocquet (2005), "Skipping stones", J.F.M., 543, 137-146.

[8] D.J. Tritton (1988), Physical Fluid Dynamics, Oxford University Press, $2^{\text {nd }}$ edition, 97-105.

[9] L.D. Landau and E.M. Lifshitz (1959), Fluid Mechanics, Pergamon Press, 168-175. 